\title{
Surgical Site Infections - Strategies for Prevention
}

\section{K.V.Rajan *}

\section{INTRODUCTION}

The term 'surgical site infection' (SSI) was introduced in 1992 to replace the previous

term 'surgical wound infection'. Surgical site infection accounts for 10 to $20 \%$ of Health Care Associated Infections (HCAI) in the UK. About 20 million surgical procedures are carried out in the US annually, out of which 600, 000 develop SSI. The delay in post-operative recovery due to SSI has economic consequences, leading to an additional cost about $£ 3000$ for each patient. It has been estimated that it requires an average additional hospital stay of 6.5 days in patients who develop SSI.

\section{Definitions Of SURGiCAL Site INFECTION (SSI)}

The Centre for Disease Prevention and Control (CDC) and the National Nosocomial Surveillance System in the US has developed standardised surveillance criteria for defining SSI.

The US Centre for Disease Control and National Nosocomial Infections Surveillance risk index is the method of risk adjustment most widely used internationally to assess the risk of developing SSI. Risk adjustment is based on three major factors:

- ASA score, reflecting the patient's state of health before surgery

- wound class, reflecting the state of contamination of the wound duration of operation, reflecting technical aspects of the surgery

\section{Strategies To Minimise The Occurrence Of Surgical Site Infections}

The interventions adopted to prevent the incidence of wound infections should be based on sound knowledge of various risk factors and the microbial flora relevant to the particular hospital setting. This includes optimisation of co-existing morbid conditions (diabetes, ischemic heart disease), cessation of smoking, judicious usage of prophylactic antibiotics and adopting guidelines for maintaining high level of asepsis during the preoperative, intra and postoperative phases.

\section{Rational Antimicrobial Prophylaxis For Preventing SSI}

Antibiotics play an important role in reducing the incidence of SSI and have the strongest evidence (level I) to support its use in surgical practice. On the other hand, antibiotic prophylaxis is never a substitute for asepsis and good surgical technique.

The antibiotic chosen should be directed against the most likely infecting organism in the particular patient, relevant to the type of surgery as well as the hospital setting.

Aims of Rational Antimicrobial therapy:

- Reduce the incidence of surgical site infection (SSI)

- Minimize the effect on the patient's normal bacterial flora.

- Minimize adverse side effects of antibiotics.

- Minimize the emergence of antibiotics resistant strains of bacteria.

- Cost effectiveness.

\section{Principles Of Prescribing Prophylactic Antibiotics}

\section{Indication}

The current recommendations are:

- Clean surgery without implants $\quad-\quad$ No antibiotics

\footnotetext{
* Dr.K.V.Rajan, Ms, FRCS,

Asst.Prof, Dept of Surgery

MGMCRI, Puducherry
} 
- Clean surgery with implants

- Clean contaminated

- Contaminated surgery

- Dirty

$\begin{array}{ll}- & \text { Prophylactic } \\ \text { - } & \text { Prophylactic } \\ \text { - } & \text { Therapeutic } \\ \text { - } & \text { Therapeutic }\end{array}$

\section{Choice of agent}

- Use narrow spectrum agent(s) when possible.

- Avoid cephalosporins, clindamycin, quinolones and co-amoxiclav whenever possible.

- Use appropriate alternatives for patients with penicillin/ beta-lactam allergy.

The antibiotics selected for prophylaxis must cover the expected pathogens for that operative site. Local antibiotic policy makers have the experience and information required to make recommendations about specific drug regimens based on an assessment of evidence, local information about resistance and drug costs.

\section{Timing of antibiotic}

The time taken for an antibiotic to reach an effective concentration in any particular tissue reflects its pharmacokinetic profile and the route of administration. Optimum timing is 30 minutes prior to skin incision. Antimicrobial cover may be sub-optimal if given $>1$ hour prior to skin incision or post-skin incision. Antibiotics should be administered in Theatre at induction of anaesthesia and given as a bolus injection where possible. Antibiotic prophylaxis in caesarean section is delayed until clamping of the cord in order to prevent the drug reaching the neonate. Prophylactic antibiotics should be administered at least 10 minutes before the application of a tourniquet to achieve appropriate tissue concentrations.

\section{Recording of antibiotic details}

The agent used and its dose should be recorded on the Anaesthetic Record Sheet or in the surgeon's Operation Record.

Specific instructions are written to stop antibiotics in the postoperative period to avoid multi-dosing.

\section{Frequency of administration}

Single dose is indicated for majority of procedures. Additional dosage may be indicated in the following:

- if shorter-acting antibiotics are used

- >1.5 litre intra-operative blood loss (re-dose following fluid replacement).

- Prolonged procedure (> 4 hours)

- Primary arthroplasty (up to 24 hours prophylaxis used)

Post-operative doses of antibiotic for prophylaxis are routinely avoided in majority of the surgical procedures.

Hospital acquired infection with pseudomonas and staphylococcus (including MRSA) are increasingly a problem worldwide. The other major problem is rapidly evolving anti-microbial drug resistance that can limit the number of effective anti-microbial agents. In this context, it would be prudent to take advice from the Institute microbiologist about the prevalent antibiotic resistance and the appropriate antibiotics to use in order to be both effective and to minimise the development of resistant strains. A local guideline outlining the rational use of antibiotics in the prevention of SSI should be formulated and this should be strictly followed by all the surgical specialities attached to the Institute.

In addition to the efforts of individual surgeons, an effective infection control program is essential in reducing Hospital-wide rates of SSI. Components of a successful program include adequate surveillance, data collection for SSI and feedback to individual surgeons to modify their current practices. Effective teamwork between anaesthetists, surgeons, nursing personnel and local microbiology department is of paramount importance to lower the impact on healthcare by SSI. 